\title{
Direct Retino-Raphe Projection Alters Serotonergic Tone and Affective Behavior
}

\begin{abstract}
Chaoran Ren ${ }^{1,2,3,10}$, Liju Luan ${ }^{1,2,3,10}$, Benson Wui-Man Lau ${ }^{4,5,6}$, Xin Huang ${ }^{1,2,3}$, Jian Yang ${ }^{4,5,6}$, Yuan Zhou ${ }^{1,2,3}$, Xihong Wu $\mathbf{u}^{3,7}$, Jie Gao ${ }^{1,2,3}$, Gary E Pickard ${ }^{8,9}$, Kwok-Fai So*,4,5,6 and Mingliang Pu*, ${ }^{*, 2,3}$

'Department of Anatomy, School of Basic Medical Sciences, Peking University, Beijing, China; ${ }^{2}$ Key Laboratory on Machine Perception (Ministry of Education), Peking University, Beijing, China; ${ }^{3}$ Key Laboratory for Visual Impairment and Restoration (Ministry of Education), Peking University, Beijing, China; ${ }^{4}$ Department of Anatomy and Research Center of Heart, Brain, Hormone and Healthy Aging, LKS Faculty of Medicine, Pokfulam, Hong Kong, China; ${ }^{5}$ The State Key Laboratory of Brain and Cognitive Sciences, The University of Hong Kong, Pokfulam, Hong Kong, China; ${ }^{6} \mathrm{GHM}$ Institute of CNS Regeneration, Jinan University, Guangzhou, China; ${ }^{7}$ Department of Machine Intelligence, Peking University, Beijing, China; ${ }^{8}$ School of Veterinary Medicine and Biomedical Sciences, University of Nebraska-Lincoln Lincoln, NE, USA; ${ }^{9}$ Department of Ophthalmology and Visual Sciences, University of Nebraska Medical Center, Omaha, NE, USA
\end{abstract}

Light is a powerful modulator of higher-order cognitive processes such as mood but it remains unclear which neural circuits mediate the impact of light on affective behavior. We found that light deprivation produces a depressive-like behavioral state that is reversed by activation of direct retinal signals to the serotonergic dorsal raphe nucleus (DRN) in a manner equivalent to treatment with the selective serotonin reuptake inhibitor fluoxetine. Surprisingly, the DRN-projecting retinal ganglion cells (RGCs) are indistinguishable from the classic alpha/Y-like RGC type that contributes to image-forming visual pathways. Silencing RGC firing or specific immunotoxin ablation of DRN-projecting RGCs increased depressive-like behavior and reduced serotonin levels in the DRN. Serotonin has a key role in the pathophysiology of depression, and these results demonstrate that retino-raphe signals modulate DRN serotonergic tone and affective behavior.

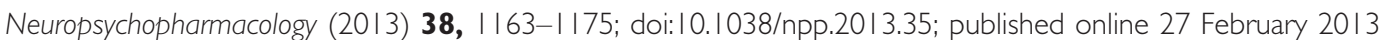

Keywords: dorsal raphe nucleus; retinal ganglion cell; depression; affective visual information; SSRI

\section{INTRODUCTION}

Photon capture by retinal photoreceptors is the initial step in visual perception (Yau and Hardie, 2009). Additionally, light stimulation of the retina is a powerful modulator of non-visual functions such as cognition, conditioned fear responses, circadian rhythms, and affective behavior (Vandewalle et al, 2009; Wirz-Justice et al, 2009; Warthen et al, 2011). Light therapy can be an effective treatment for both seasonal and non-seasonal mood disorders (Lewy et al, 1987; Golden et al, 2005) while depressive-like behavior can be induced in mammals across species by light deprivation (Wilson, 2002; Gonzalez and Aston-Jones, 2008; Monje et al,

*Correspondence: Professor K-F So, LKS Faculty of Medicine, 21 Sassoon Road, Pokfulam, Hong Kong, China, Tel: +852 2819 9216, Fax:+852 2817 6821, E-mail: hrmaskf@hku.hk

Professor M Pu, Anatomy/Embryology, School of Basic Medical Sciences; Key Laboratory on Machine Perception; Key Laboratory for Visual Impairment and Restore, Peking University, 38 Xueyuan Road, Beijing I00|9|, China, Tel: +86I0 8280 2972, Fax +86I0 82802877 , E-mail: mpu@hsc.pku.edu.cn

${ }^{10}$ These authors contributed equally to this work.

Received 8 August 2012; revised 24 December 2012; accepted 28 January 2013; accepted article preview online 31 January 2013
2011; Lau et al, 2011). Our past study showed that alpha/Y-like retinal ganglion cells (RGCs) innervate the midbrain dorsal raphe nucleus (DRN; Luan et al, 2011) and the DRN has a crucial role in regulating affective behavior. Recent evidence shows that a group of intrinsically photosensitive RGCs (ipRGCs) is responsible for processing nonimage-forming visual functions (Güler et al, 2008). However, it remains to be defined whether ipRGCs or conventional RGCs contribute to affective behavior. Here we show that alpha/Y-like RGCs with no apparent intrinsic photosensitivity send signals to serotonergic neurons of the midbrain DRN mediating the effects of light both on depressive-like behavior and DRN serotonin (5-HT) levels. Moreover, we demonstrate that the effect of RGC input to the DRN is similar to that of selective serotonin reuptake inhibitor (SSRI) treatment on depressive-like behavior.

\section{MATERIALS AND METHODS}

All experiments were performed in accordance with Peking University guidelines for animal research and the Association for Research in Vision and Ophthalmology (ARVO) Statement for the Use of Animals in Ophthalmic and Vision Research. The experimental animal protocol was 
approved by Peking University Institutional Animal Care and Use Committee (IACUC).

\section{Animals}

Male adult Mongolian gerbils (2-3-months old, 65-77g) were used in these experiments. Upon arrival all animals were maintained in 12L : 12D conditions (LD) with food and water provided ad libitum. For light-deprivation experiments, all conditions were the same except animals were kept in complete darkness (DD) for 14 days.

\section{Stereotaxic Surgeries}

Cholera toxin B subunit (CTB)-conjugated Alexa Fluor 488 or 594 (C34777 or C22842, Invitrogen Inc., Grand Island, NY) was deposited into the DRN or the superior colliculus (SC) as described previously (Luan et al, 2011). Briefly, animals were anesthetized with $60 \mathrm{mg} / \mathrm{kg}$ sodium pentobarbital by intraperitoneal injection (IP) and placed in a stereotaxic device (Stoelting, Wood Dale, IL). After a craniotomy was performed, a Hamilton syringe was inserted into the DRN or SC and $1.5 \mu \mathrm{l}$ of CTB-conjugated Alexa Fluor was injected, the wound was sutured, and 3 days were allowed for retrograde transport of CTB to the retina.

\section{Immunocytochemistry and Image Processing}

All animals were anesthetized $(60 \mathrm{mg} / \mathrm{kg}$ sodium pentobarbital, IP) and perfused intracardially with $0.9 \%$ saline followed by $4 \%$ paraformaldehyde in phosphate-buffered saline (PBS). Brains and eyes were removed.

Detection of melanopsin-expressing RGCs was performed using immunocytochemical procedures on retinal whole mounts. Retinas were placed in $0.1 \mathrm{M}$ PBS containing $10 \%$ normal goat serum (Vector Laboratories, Burlingame, CA) with $0.3 \%$ Trition-X-100 (T8787, Sigma-Aldrich, St Louis, MO) for $1 \mathrm{~h}$ before incubation for 3 days at $4{ }^{\circ} \mathrm{C}$ in a rabbit anti-melanopsin antibody diluted 1:600; antibody was generated against an $\mathrm{N}$-terminal amino-acid sequence of melanopsin protein (PA1-780, Fisher Scientific Inc., Barrington, IL). This was followed by incubation with a secondary antibody (Dylight488 or Dylight549) goatanti-rabbit IgG $(1: 200$, Vector Laboratories).

Immunocytochemical staining of serotonergic neurons was performed on free-floating brain sections. Briefly, $40-\mu \mathrm{m}$ thick cryostat sections collected through the DRN were placed in $0.1 \mathrm{M}$ PBS containing $10 \%$ normal goat serum with $0.3 \%$ Trition-X-100 for $1 \mathrm{~h}$ before incubation in rabbit anti-serotonin antibody (1:500, Sigma-Aldrich) for $72 \mathrm{~h}$ at $4{ }^{\circ} \mathrm{C}$. Brain sections were then placed in secondary antibody Dylight488 goat-anti-rabbit $\operatorname{IgG}(1: 200$, Vector Laboratories) for $6 \mathrm{~h}$ at room temperature.

The method for converting intracellularly injected Lucifer Yellow (Sigma-Aldrich) into a permanent dye has been described previously ( $\mathrm{Pu}, 1999)$. Briefly, after the completion of electrophysiological recording, retinas containing DRN-projecting RGCs intracellularly injected with Lucifer Yellow were incubated in $4 \%$ paraformaldehyde in $0.1 \mathrm{M}$ PBS for $2 \mathrm{~h}$. Retinas were rinsed in $0.1 \mathrm{M} \mathrm{PBS}$ for $10 \mathrm{~min}$ three times and placed in $10 \%$ normal goat serum containing $2 \%$ Trition-X-100 and $0.5 \%$ dimethyl sulfoxide (Sigma-Aldrich) for $48 \mathrm{~h}$ at $4{ }^{\circ} \mathrm{C}$. Retinas were then incubated in rabbit-anti-Lucifer Yellow antibody $(1: 1000$, Sigma-Aldrich) for $48 \mathrm{~h}$ at $4{ }^{\circ} \mathrm{C}$ followed by secondary antibody Dylight 549 goat-anti-rabbit IgG $(1: 200$, Vector Laboratories) for $6 \mathrm{~h}$ at room temperature. All sections and retinas were rinsed in $0.1 \mathrm{M}$ PBS and cover-slipped in anti-fading aqueous mounting medium (EMS, Hatfield, PA).

\section{Confocal Microscopy and Three-Dimensional Reconstruction}

RGCs injected with Lucifer Yellow and immunocytochemically stained were scanned with a confocal microscope (TCS SP5 II, Leica Microsystems, Wetzlar, Germany). The Z axis interval was $0.2 \mu \mathrm{m}$. Each stack of optical sections covered a retinal area of $325.75 \times 325.75 \mu \mathrm{m}^{2}(1024 \times 1024$ pixels $)$. Using Image J and Photoshop CS5 (Adobe Corp., San Jose, California), each stack of optical sections was arranged as a montage and projected to a $0^{\circ} \mathrm{X}-\mathrm{Y}$ plane and a $90^{\circ} \mathrm{Y}-\mathrm{Z}$ plane to obtain a three-dimensional reconstruction of the cell. Details of three-dimensional reconstruction and confocal calibration procedures are described elsewhere (Pu, 1999).

\section{Selective Elimination of Retinal Photoreceptors}

To selectively eliminate retinal rod and cone photoreceptors, animals received a single IP injection of $\mathrm{N}$-methyl$\mathrm{N}$-nitrosourea (MNU; N1517, Sigma-Aldrich). MNU is a direct-acting alkylating agent that targets photoreceptors causing rapid apoptosis while sparing RGCs (Herrold, 1967; Wenzel et al, 2000; Yoshizawa and Tsubura, 2005; Boudard et al, 2009). MNU was dissolved in saline with the final concentration of $10 \mathrm{mg} / \mathrm{ml}$ and the injected dosage was $80 \mathrm{mg} / \mathrm{kg}$ body weight, a dose in the range of MNU used in other species (Yoshizawa and Tsubura, 2005). This dosage was chosen specifically based on a pilot study in which a dose was determined that could effectively eliminate photoreceptors without affecting the health status of animals. MNU-treated animals were returned to their home cages and maintained under LD conditions for at least 7 days before they were assigned to an experimental group.

\section{Selective Targeting of ipRGCs, SC-projecting RGCs, DRN-projecting RGCs and Blocking Retinal Spiking Activity}

We adopted the immunotoxin method described by Göz et al (2008) to selectively destroy melanopsin-expressing ipRGCs. Briefly, under deep anesthesia, animals $(n=6 /$ group) received intravitreal injections of anti-melanopsinsaporin conjugate $(2 \mu \mathrm{g} / \mathrm{eye}$, \#IT-44, Advanced Targeting Systems, San Diego, CA) using a 5- $\mu$ l Hamilton syringe fitted with a $30-G$ needle. The syringe was held in place for 2 min after immunotoxin delivery and gradually withdrawn over the next $60 \mathrm{~s}$. Animals were returned to their home cage for the next 14 days.

To selectively remove SC-projecting RGCs, animals that had received CTB-conjugated Alexa Fluor 488 ( $1.5 \mu \mathrm{l} /$ animal) deposited into the SC 3 days earlier, received intravitreal injection of a custom conjugate made between saporin (\#IT-27-100, Advanced Targeting Systems) and an 
affinity-purified anti-CTB antibody (GCHL-55A-Z, Immunology Consultants Laboratory, Portland, OR). Animals $(n=6$ /group) received bilateral intravitreal injections of different quantities ( $0 \mu \mathrm{g}, 0.5 \mu \mathrm{g}, 2 \mu \mathrm{g}, 4 \mu \mathrm{g})$ of the immunotoxin and were returned to their home cage for 14 days.

To selectively remove DRN-projecting RGCs, animals that had received CTB-conjugated Alexa Fluor 594 ( $1.5 \mu \mathrm{l} /$ animal) deposited in the DRN 3 days earlier, received intravitreal injections ( $2 \mu \mathrm{g} /$ eye $n=6 /$ group $)$ of the same immunotoxin used for the ablation of SC-projecting RGCs described above. This dose was chosen based on the results of a pilot study in which a dose was determined that could effectively eliminate the CTB-labeled SC-projecting RGCs. After intravitreal injection, animals were returned to their cages for at least 14 days before being assigned to an experimental group.

To block the action potentials produced by RGCs in MNU-treated animals, we adopted a tetrodotoxin (TTX) intravitreal injection protocol described by other groups (Edwards and Cline, 1999; Viswanathan et al, 2000; Witkovsky et al, 2004). TTX is a potent voltage-gated sodium channel blocker that blocks sodium-dependent action potentials in RGCs and prevents transmission of visual signals from retina. TTX (1069, Tocris Bioscience, Ellisville, MO) was prepared as $3 \mathrm{mM}$ in distilled water; $6 \mu \mathrm{M}$ TTX in a volume of $5 \mu \mathrm{l}(5 \mu \mathrm{l}$ saline serves as vehicle, $n=6 /$ group) was injected into the vitreous chamber of each eye using a 30-G needle attached to a 5- $\mu$ l Hamilton syringe. After injections, animals were returned to their home cage for $90 \mathrm{~min}$ before they were assigned to behavioral tests.

\section{Chronic Treatment with Anti-depressants}

The light-deprived (DD) animals received daily IP injections of fluoxetine $(10 \mathrm{mg} / \mathrm{kg}, n=6)$, imipramine $(10 \mathrm{mg} / \mathrm{kg}$, $n=6$ ), or vehicle (saline, $400 \mu \mathrm{l}, n=6$ ) for 21 days. The dosage for chronic treatment with anti-depressants was determined based on previous publications (Detke et al, 1996; Bijak and Papp, 1995; Page et al, 1999; Svenningsson et al, 2002; Rodríguez-Gaztelumendi et al, 2009; Machado et al, 2012). Fluoxetine hydrochloride (F132, Sigma-Aldrich) and imipramine hydrochloride (I7379, Sigma-Aldrich) were dissolved in saline with a final concentration of $2 \mathrm{mg} / \mathrm{ml}$. Daily injections were delivered at 1700 hours under dim red light (5-9 lux), except on testing days. To minimize possible peritoneal irritation, IP injection sites were alternated between left and right lower abdominal quadrants on a daily basis. After injection, animals were returned to their home cages and kept in complete darkness.

\section{Physiological Recording and Data Analysis}

DRN-projecting RGCs were recorded as previously described (Luan et al, 2011). Briefly, the eyes were enucleated under dim red light; lens and vitreous were carefully removed with a pair of fine forceps. The eyecup was flat-mounted, sclera side down, directly on the bottom of a recording chamber, and was superfused by oxygenated $\left(95 \% \mathrm{O}_{2} / 5 \% \mathrm{CO}_{2}\right)$ Ames medium (Sigma-Aldrich) at a fixed rate $(5 \mathrm{ml} / \mathrm{min})$ at room temperature between $22-24^{\circ} \mathrm{C}$. Visual stimuli were generated by programming the
Psychophysics Toolbox in Matlab displayed on a Samsung mini LED projector (Samsung SP-P310ME, Samsung Electronics Co Ltd, Suwon City, Korea) and imaged with a first-surface mirror and lens (Edmond Scientific, Barrington, NJ) on the film plane of the microscope's camera port. The luminance level of the projector was measured with a digital radiometer (S370 Radiometer, UDT Instruments, San Diego, CA) using a $\times 40$ water-immersion objective (Carl Zeiss, Thornwood, NY). The irradiance was further reduced using neutral density filters (Oriel Corp., Stratford, CT). Visual responses and spontaneous activity of DRNprojecting RGCs were recorded using a glass microelectrode and amplified with a patch clamp amplifier (Multiclamp 700B) and digitized (Digidata 1430; Axon Instrument, Inc., Forest City, CA). RGCs retrogradely labeled by CTB were targeted for recording. To test the blocking effect of TTX on the action potentials of DRN-projecting RGCs, $20 \mathrm{ml}$ oxygenated $\left(95 \% \mathrm{O}_{2} / 5 \% \mathrm{CO}_{2}\right)$ Ames medium containing $0.1 \mu \mathrm{M}$ TTX was superfused into the chamber $5 \mathrm{~min}$ before cell recording. The concentration of TTX was determined based on the work of Margolis and Detwiler (2007). Once the physiological profiling was completed, recorded RGCs were intracellularly filled with Lucifer Yellow for morphological evaluation. The acquired physiological data were further analyzed off-line (pCLAMP9; Axon Corp., CA).

\section{Modified Forced Swimming Test (FST)}

Modified forced swimming tests (FST) were conducted as previously described (Lau et al, 2011). Briefly, animals in each experimental group were tested in two trials. In the first trial, animals were forced to swim in a glass cylinder (silicon dioxide, diameter: $30 \mathrm{~cm}$, height: $50 \mathrm{~cm}$, water depth: $30 \mathrm{~cm}$, water temperature: $28^{\circ} \mathrm{C}$ ) for $15 \mathrm{~min}$. Then animals were dried and returned to their cages for $24 \mathrm{~h}$. The second trial lasted for $5 \mathrm{~min}$. The entire procedure was recorded with an infrared camera placed above the glass cylinder. Three featured behaviors during the second session were measured: swimming (active movement that crossed at least one quadrant of the cylinder); climbing (pulling movements of forepaws that scratched at the wall of cylinder); and immobility (no active movement except that needed to keep the animal from drowning). A behaviorsampling technique (Cryan et al, 2005a) was used to score movements as defined above, which measure the frequency of behaviors during $5 \mathrm{~s}$. To observe any time-of-day influence on animal's affective behavior, animals in the LD, DD, MNU-treated (MNU-LD and MNU-DD) groups ( $n=6$ /group at each time point) were tested at four different Zeitgeber Time (ZT) points (ZT0, ZT6, ZT12, $\mathrm{ZT} 18)$ where $\mathrm{ZT} 0=$ light onset and $\mathrm{ZT} 12=$ light offset in LD. Animals in the other groups were tested at ZT6. To minimize the effect of light on the affective behavior of animals, each trial was conducted under dim red light (25lux).

\section{Tail Suspension Test (TST)}

Depression-like behavior of animals was assayed using the tail suspension test (TST), which is widely used for testing behavioral despair of rodents, including the gerbil 
(Steru et al, 1985; Varty et al, 2003). Briefly, the animal, held by the tail, was suspended $50 \mathrm{~cm}$ from the floor and the behavior of the animal was recorded by a video camera for $6 \mathrm{~min}$. The immobile time during the last $4 \mathrm{~min}$ was counted. Each test was performed at ZT6 under dim red light (25lux).

\section{Open Field Test (OFT)}

Motor activity was measured in an open field test box $(74 \times 74 \times 40 \mathrm{~cm})$. A $4 \times 4$ grid was superimposed onto the floor to score the locomotor activity. This experiment included two sessions. In the first, each animal was allowed to explore in the box for $3 \mathrm{~min}$ to reduce gerbil's 'defensive convulsions in response to novel environment' (Pickles et al, 2012). Then each animal was returned to its home cage. The second session was performed $24 \mathrm{~h}$ later. Animals in each group were placed in the center of the open field and left to explore for $60 \mathrm{~min}$. All animal activity was recorded with an infrared camera placed above the box. The distance traveled by each animal during the second test session was measured. Each test started at ZT6 under dim red light (25lux). The box was wiped clean with a paper towel soaked in $50 \%$ ethanol and dried thoroughly between each test session.

\section{Sucrose Preference Test (SPT)}

Animals were tested for preference of a $2 \%$ sucrose solution (Sucrose, Sigma-Aldrich), using a two-bottle choice procedure as described by Monteggia et al (2007) with slight modifications. Each animal was housed individually during the 2-day test period. Animals were given two bottles, one of sucrose (2\%) and one of tap water. Every $24 \mathrm{~h}$ the amount of sucrose and water consumed was recorded. To prevent potential location preference of drinking, the position of the bottles was changed every $24 \mathrm{~h}$. Food and water were available ad libitum prior to the SPT. The preference for the sucrose solution was determined as the percentage of sucrose solution ingested relative to the total intake.

\section{Statistical Analysis}

Assignment of subjects to various experimental groups was random. Immobile scores at four different time points (Figure 1a) were analyzed by a two-way ANOVA test (with the two factors being type and time points) followed by Tukey's post hoc test if necessary. Other behavioral data and cell/spikes counting data were analyzed through the one-way ANOVA test followed by Tukey's post hoc test if necessary. To determine whether the level of serotonin in DRN and the forced swimming test are related, a possible correlation between the number of 5-HT + neurons in DRN and the score of three different movements in forced swimming test was explored (Pearson's test). Data are expressed as the mean \pm SEM. Statistical significance was set as $p<0.05$.

\section{Experimental Groups:}

LD: Animals were housed in $12 \mathrm{~L}: 12 \mathrm{D}(\mathrm{L} \approx 300 \mathrm{lux} ; \mathrm{D}=0$ lux) for 2 weeks.

DD: Animals were transferred from LD into complete darkness for 2 weeks.
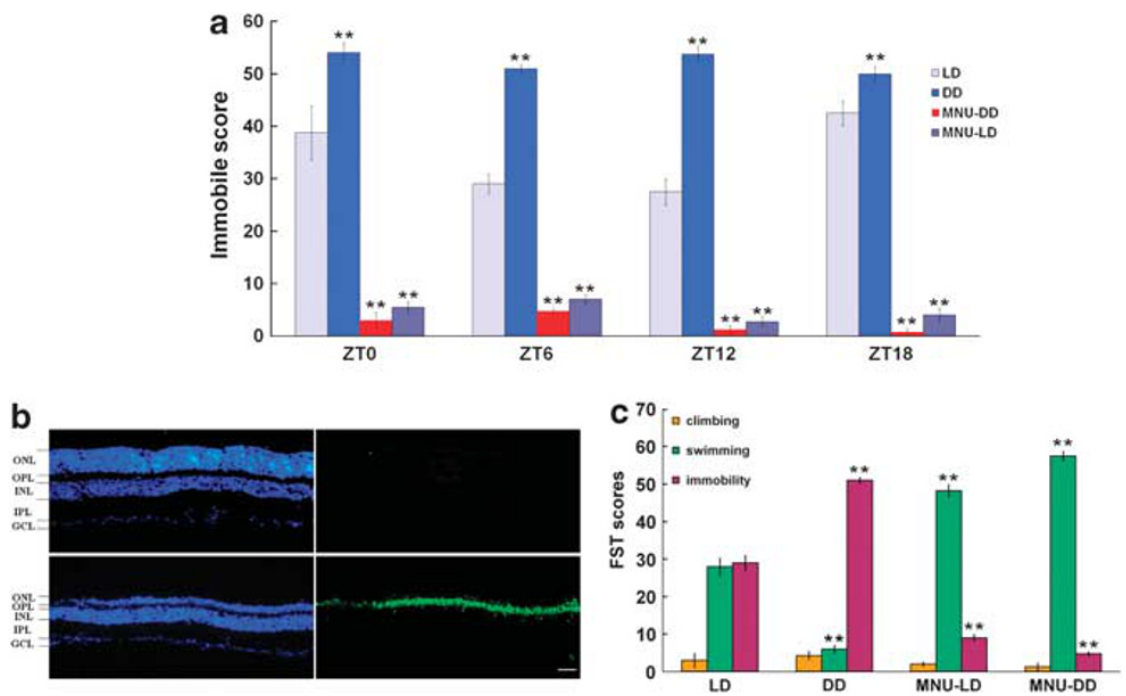

Figure I The effects of continuous darkness (DD) and photoreceptor ablation (MNU) on forced swimming test (FST)-associated behaviors. (a) Immobile score from the FST in I2-h light: I2-h dark (LD) and DD conditions, and after MNU treatment (MNU-LD and MNU-DD). Zeitgeber Time (ZT) $0=$ light onset in LD; animals in DD tested at ZT of previous LD cycle (mean \pm SEM, $n=6 /$ group/ZT). DD elevated immobile score vs LD at each ZT; MNU reduced immobile score at all ZTs vs LD or DD. Two-way ANOVA, Tukey's post-hoc test; *** $<0.00$ I. (b) Immunohistochemically stained retinal sections from untreated (top) and MNU-treated (bottom) animals. DAPI stain (left) illustrating retinal cellular layers: outer nuclear layer: ONL; outer plexiform layer: OPL; inner nuclear layer: INL; inner plexiform layer: IPL; ganglion cell layer: GCL. TUNEL stain (right) illustrating absence of apoptosis in untreated animal (top right) and apoptotic programmed cell death and photoreceptor depletion in the ONL after MNU treatment (bottom right). Bar $=20 \mu \mathrm{m}$. (c) FST climbing, swimming, and immobility scores at ZT6 for controls and MNU-treated animals. Immobile score values are taken from ZT6 in (a); $(n=6 / g r o u p)$. DD elevated immobile score and decreased swimming score vs LD; MNU decreased immobile score and elevated swimming score vs DD. Climbing scores did not differ significantly among groups. One-way ANOVA, Tukey's post-hoc test; ${ }^{*} * p<0.001$. 
MNU-DD: Animals received a single IP injection of MNU $(80 \mathrm{mg} / \mathrm{kg})$ while in LD conditions. One week later, animals were transferred to DD for 2 weeks.

MNU-DD-TTX: After being housed under DD for 2 weeks, MNU-treated animals received an intravitreal injection of tetrodotoxin (TTX; $6 \mu \mathrm{M} /$ eye) using a $30-\mathrm{G}$ needle attached to a 5- $\mu$ l Hamilton syringe. FST and TST were conducted 90 min after delivery of TTX.

MNU-DD-VEH-1: MNU-treated animals in DD conditions received an intravitreal injection of saline $(5 \mu \mathrm{l} / \mathrm{eye})$. These animals were then housed under DD for $90 \mathrm{~min}$.

MNU-DD-anti-Mel-SAP: MNU-treated animals housed in LD conditions received an intravitreal injection of melanopsin antibody-saporin conjugate $(2 \mu \mathrm{g} / \mathrm{eye})$. These animals were then housed under DD for 2 weeks.

MNU-DD-VEH-2: MNU-treated animals housed in LD conditions received an intravitreal injection of saline ( $5 \mu \mathrm{l} /$ eye). These animals were then housed under DD for 2 weeks.

DD-VEH-1: Animals were transferred from LD into DD for 2 weeks. Then each animal received intravitreal injection of saline $(5 \mu \mathrm{l} / \mathrm{eye})$. These animals were then housed under DD for 2 weeks.

MNU-DD-anti-CTB-SAP: MNU-treated animals were anesthetized $(60 \mathrm{mg} / \mathrm{kg}$ sodium pentobarbital, IP) and placed in a stereotaxic apparatus. After a craniotomy was performed, a Hamilton syringe was inserted and stereotaxically positioned in the DRN and CTB $(1.5 \mu \mathrm{l} /$ animal $)$ was deposited into the DRN. Three days after CTB injection, each animal was intravitreally injected with antiCTB-saporin conjugate $(2 \mu \mathrm{g} / \mathrm{eye})$. They were housed in DD for 2 weeks after intravitreal injections.

MNU-DD-VEH-3: MNU-treated animals were anesthetized $(60 \mathrm{mg} / \mathrm{kg}$ sodium pentobarbital, IP) and placed in a stereotaxic apparatus. After a craniotomy was performed, a Hamilton syringe was inserted and stereotaxically positioned in the DRN and CTB $(1.5 \mu \mathrm{l} / \mathrm{animal})$ was deposited into the DRN. Three days after CTB injection, each animal was intravitreally injected with saline $(5 \mu \mathrm{l} /$ eye). They were housed in DD for 2 weeks after intravitreal injections.

LD-anti-CTB-SAP: animals housed in LD conditions were anesthetized $(60 \mathrm{mg} / \mathrm{kg}$ sodium pentobarbital, IP) and placed in the stereotaxic apparatus as described above. After a craniotomy was performed, a Hamilton syringe was inserted and stereotaxically positioned in the DRN and CTB (1.5 $\mu \mathrm{l} /$ animal) was deposited into the DRN. Three days after CTB injection, each animal was intravitreally injected with anti-CTB-saporin conjugate $(2 \mu \mathrm{g} /$ eye $)$. They were housed in LD for 2 weeks after intravitreal injections.

LD-VEH: animals housed in LD conditions were anesthetized $(60 \mathrm{mg} / \mathrm{kg}$ sodium pentobarbital, IP) and placed in the stereotaxic apparatus. After a craniotomy was performed, a Hamilton syringe was inserted and stereotaxically positioned in the DRN and CTB $(1.5 \mu \mathrm{l} / \mathrm{animal})$ was deposited into the DRN. Three days after CTB injection, each animal was intravitreally injected with saline $(5 \mu \mathrm{l} /$ eye). They were housed in LD for 2 weeks after intravitreal injections.

DD-FLX: Animals were transferred from LD to complete darkness for 2 weeks. Then each animal received IP injections of fluoxetine $(10 \mathrm{mg} / \mathrm{kg} /$ day $)$ for 3 weeks.

DD-IMI: Animals were transferred from LD to complete darkness for 2 weeks. Then each animal received IP injections of imipramine hydrochloride $(10 \mathrm{mg} / \mathrm{kg} /$ day $)$ for 3 weeks.

DD-VEH-2: Animals were transferred from LD to complete darkness for 2 weeks. Then each animal received IP injections of saline $(0.4 \mathrm{ml} /$ day $)$ for 3 weeks.

\section{RESULTS}

\section{The Effects of Continuous Darkness (DD) and Photoreceptor Ablation (MNU) on Behavioral State}

Animals in DD exhibit significantly elevated immobile scores compared with animals housed in LD conditions, supporting the interpretation that light deprivation is associated with a depressive-like behavioral state (LD vs DD at four time points, two-way ANOVA, effect of type $\times$ time points interaction: $\mathrm{F}_{3,40}=12.86, \mathrm{p}<0.001$; effect of type: $\mathrm{F}_{1,40}=407.95, \quad p<0.001$; Turkey's post hoc comparison indicated that immobile score in DD group was significantly higher than in LD group at each time point, $p<0.001$; Figure 1a). Results from both the TST (Figure 2a) and the SPT (Figure 3a) reveal that DD induced depressive-like behavior (TST: one-way ANOVA, $\mathrm{F}_{1,10}=463.12, p<0.001$; SPT: one-way ANOVA, $\mathrm{F}_{1,10}=16.15, p<0.01$ ), while results from the OFT (Figure 4) indicate that the DD-induced change in behavior was not associated with an alteration in locomotor activity (one-way ANOVA, $\mathrm{F}_{6,28}=0.92, p=0.49$ ).

As described earlier, to begin to define the neural circuits by which light deprivation provokes depressive-like behavior, rod and cone photoreceptor degeneration was induced by a single IP injection of MNU, a direct-acting alkylating agent that causes rapid photoreceptor apoptosis. MNU-treated animals lacking rods and cones (Figure $1 \mathrm{~b}$ ) demonstrated a dramatic reduction in immobile score compared with untreated animals in LD conditions (LD vs MNU-LD at four time points, two-way ANOVA, effect of type $\times$ time points interaction: $\mathrm{F}_{3,40}=23.44, p<0.001$; effect of type: $\mathrm{F}_{1,40}=1374.35, \quad p<0.001$; Turkey's post hoc comparison indicated that immobile score in MNU-LD group was significantly lower than in LD group at each time point, $p<0.001$ ) or after 14 days in DD (DD $v s$ MNU-DD at four time points, two-way ANOVA, effect of type $\times$ time points interaction: $\mathrm{F}_{3,40}=1.3, p=0.287$; effect of type: $\mathrm{F}_{1,40}=4790.33, p<0.001$; Figure 1a).

\section{DRN-projecting RGC Activity Regulates Behavioral Mood}

Photoreceptor degeneration causes a striking increase in the rate of OFF RGC spontaneous spike discharge ( $\mathrm{Pu}$ et al, 2006; Margolis and Detwiler, 2007). To determine whether MNU-induced photoreceptor apoptosis increased RGC spontaneous activity, single-unit recordings were made from alpha/Y-like DRN-projecting OFF RGCs (Figure 5a). These cells showed vigorous spontaneous activity compared with alpha/Y-like DRN-projecting OFF RGCs in intact retinas (one-way, ANOVA, $\mathrm{F}_{1,18}=380.05$, $p<0.001$; Figure $5 b-c)$, which was completely silenced by application of tetrodotoxin (TTX), a potent voltage-gated sodium channel blocker (Figure $5 \mathrm{~b}$ ). Thus, as in other models of degenerative photoreceptor loss, MNU-induced 

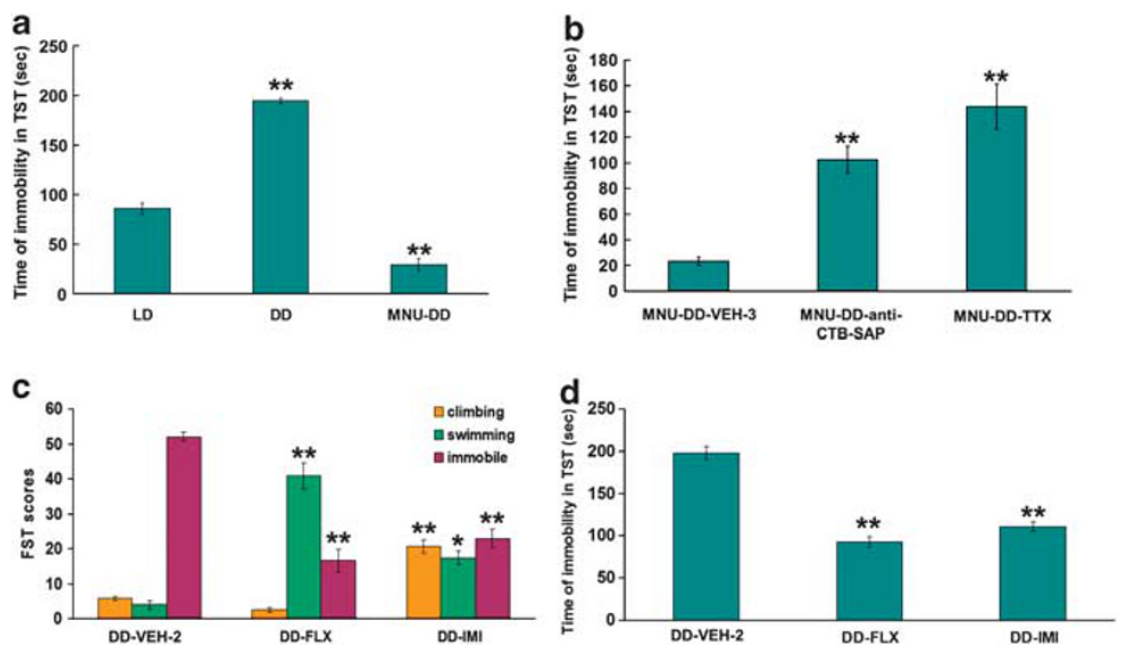

Figure 2 Time of immobility under different testing conditions and effects of chronic treatment with fluoxetine (FLX) and imipramine hydrochloride (IMI). (a) Time of immobility of the 12-h light, 12-h dark (LD), continuous darkness (DD), and photoreceptor-ablation-DD (MNU-DD) groups measured using the tail suspension test (TST). LD group serves as control (one-way ANOVA; *** $<<0.00 \mathrm{I}$ ). (b) Time of immobility of the MNU-DD-vehicle (MNU-DD-VEH-3), MNU-DD-anti-CTB-saporin immunotoxin (MNU-DD-anti-CTB-SAP), and MNU-DD-tetrodotoxin (MNU-DD-TTX) groups measured through TST, MNU-DD-VEH-3 group serves as control (one-way ANOVA; ** $<0.00$ I). (c) Forced Swimming test (FST) climbing, swimming, and immobility scores for saline (VEH), FLX, and IMI chronic treatment. DD-VEH-2 group serves as control. One-way ANOVA; * $p<0.05$; *** $<0.00$ I. (d) The effect of FLX and IMI chronic treatment on immobility during TST. DD-VEH-2 group serves as control. One-way ANOVA; *** $<0.001$.
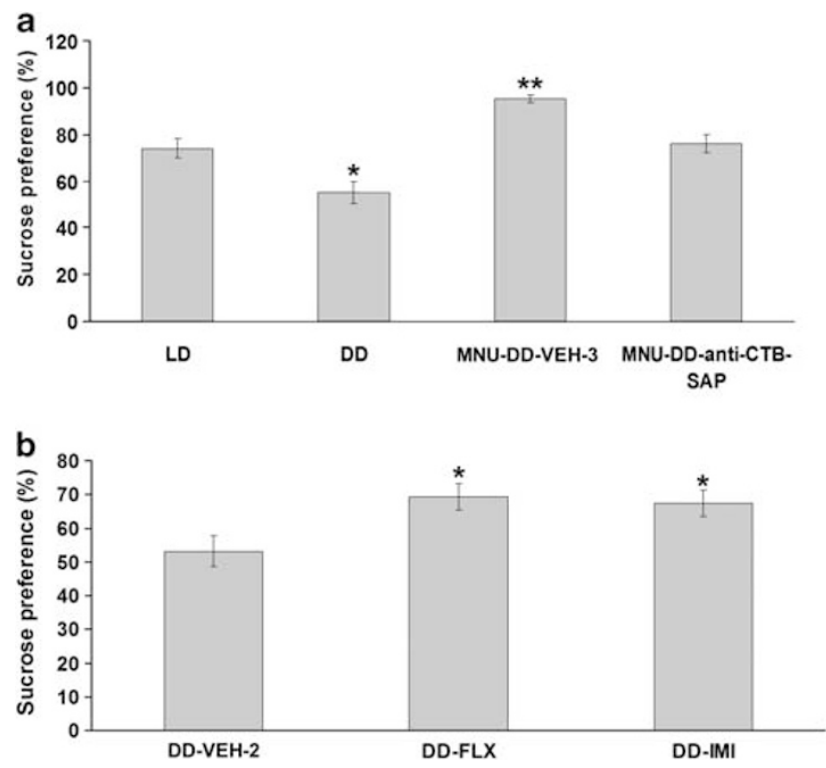

Figure 3 Anhedonia demonstrated using the sucrose preference test (SPT). (a) Sucrose preference of animals in the 12-h light, 12-h dark (LD), continuous darkness (DD), photoreceptor ablation-DD-vehicle (MNU-DD-VEH-3), and MNU-DD-anti-cholera toxin subunit B saporin immunotoxin (MNU-anti-CTB-SAP) groups. Animals had decreased sucrose preferences after light deprivation, which could be reversed by MNU treatment. Sucrose preference of animals in MNU-DD group decreased significantly after intravitreal anti-CTB-SAP treatment. LD group serves as control. One-way ANOVA; $* p<0.05$; *** $<0.00$ I. (b) Sucrose preference of animals in the DD-VEH-2, DD-fluoxetine (DD-FLX), and DD-imipramine hydrochloride (DD-IMI) groups. Sucrose preference of animals in the DD group elevated significantly after FLX/IMI chronic treatment. DD-VEH-2 group serves as control. One-way ANOVA; $* p<0.05$. Total intake of liquid (tap water plus sucrose solution) was unchanged between the groups (one-way ANOVA, $F_{5,24}=0.58, p=0.72$; Supplementary Figure S4). Results are presented as mean of sucrose preference (the percentage of sucrose solution ingested relative to the total amount of liquid consumed) \pm SEM.

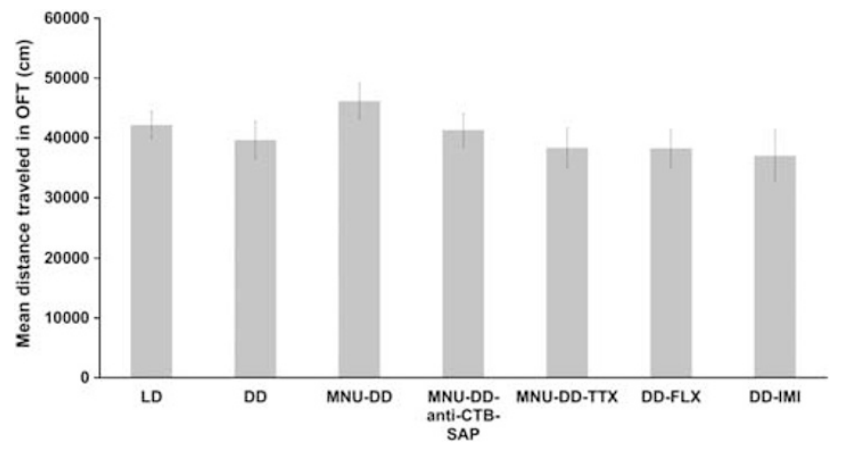

Figure 4 Locomotor activity assessed using open field test (OFT). The histogram shows mean distance traveled by animals under different experimental conditions, ie, 12-h light, I2-h dark (LD), continuous darkness (DD), photoreceptor ablation-DD (MNU-DD), MNU-DD-anti-cholera toxin subunit $B$ saporin immunotoxin (MNU-DD-anti-CTB-SAP), MNU-DD-tetrodotoxin (MNU-DD-TTX), DD-fluoxetine (DD-FLX), and DD-imipramine hydrochloride (DD-IMI) during a 60 min test. DD group serves as control. Results are presented as mean of distance traveled in $\mathrm{cm} \pm \operatorname{SEM}$ ( $n=5$ animals/group).

rod/cone degeneration provoked a high rate $(30 \mathrm{~Hz})$ of sustained spontaneous firing of OFF RGCs.

To test whether the increase in OFF RGC spontaneous activity was responsible for the dramatic immobile score decline in MNU-DD animals, additional MNU-DD animals were examined with the FST 90 min after RGC spontaneous activity was blocked by in vivo intravitreal TTX application. The short-term inhibition of RGC firing significantly increased immobile score (one-way ANOVA, $F_{1,10}=$ 250.43, $p<0.001)$ and decreased swimming in MNU-DDTTX animals compared with controls (one-way ANOVA, $\mathrm{F}_{1,10}=204.96, p<0.001$; Figure $5 \mathrm{~d}$ ). 
a

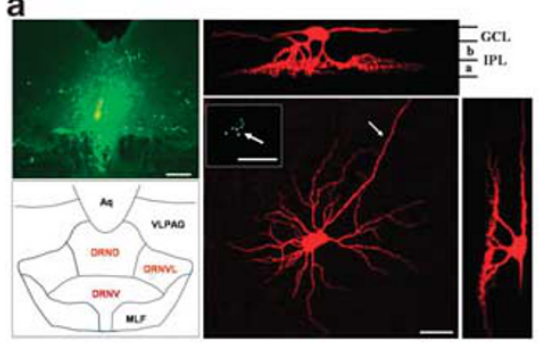

C

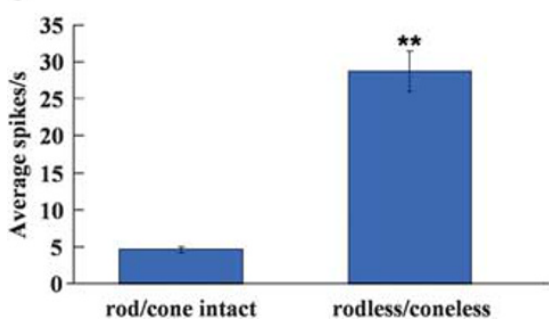

b
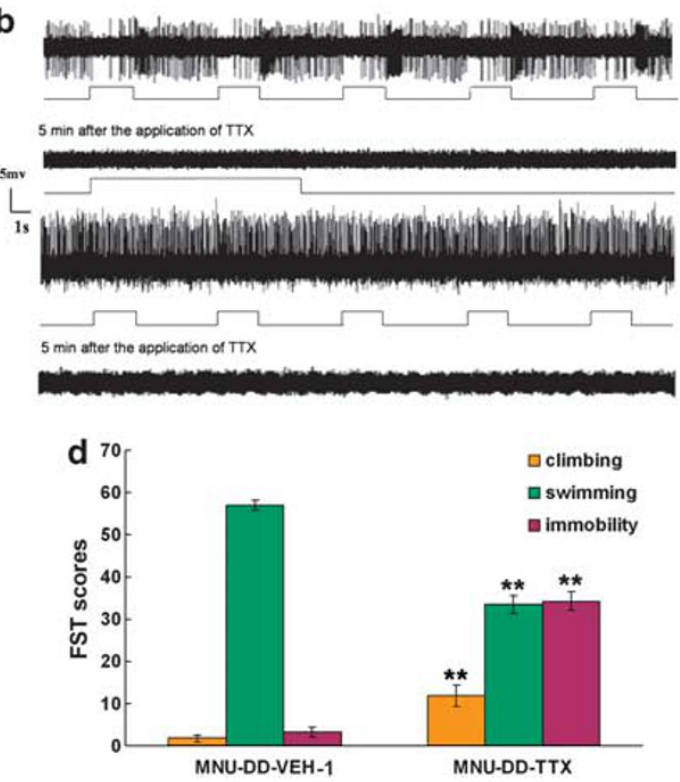

Figure 5 Dorsal raphe nucleus (DRN)-projecting retinal ganglion cells (RGCs) activity regulates behavior. (a) Top left: CTB-conjugated Alexa Fluor injected into DRN labels cell bodies of DRN-projecting RGCs. Bottom left: A schematic of DRN region. Aqueduct: Aq; ventrolateral periaqueductal gray: VLPAG; dorsal raphe nucleus, dorsal: DRND; dorsal raphe nucleus, ventral: DRNV; dorsal raphe nucleus, ventrolateral: DRNVL; medial longitudinal fasciculus: MLF. Modified from Paxinos and Watson (1998). Bottom right: confocal 3D reconstruction of a DRN-projecting RGC. Arrow in inset at upper left corner points to the retrogradely labeled soma. After recording and filling, the image of the cell clearly reveals typical alpha RGC morphology (large soma, radially oriented dendrites, large dendritic field (Peichl et al, 1987); arrow indicates axon). Bars $=200 \mu \mathrm{m}$, top left; $20 \mu \mathrm{m}$, insert; $50 \mu \mathrm{m}$, bottom right. Images of the neuron in bottom right rotated $90^{\circ}$ illustrate dendrites in the OFF (sublamina a) of the inner plexiform layer (IPL). OFF sublamina a: a; ON sublamina b: b; GCL: ganglion cell layer. (b) Firing patterns of DRN-projecting OFF alpha RGCs in response to visual stimulation. Top trace: Transient discharge pattern of retrogradely labeled OFF Y-like RGC (firing increases at light offset). Second trace from top: Loss of light-evoked firing 5 min after application of $0.1 \mu \mathrm{M}$ of the voltage-gated sodium channel blocker, tetrodotoxin (TTX). Third trace: OFF alpha DRN-projecting RGC from MNU-treated retina illustrating loss of visual responsiveness and vigorous spontaneous firing. Bottom trace: Loss of spontaneous firing 5 min after $0.1 \mu M$ TTX application. (c) Spontaneous discharge rate of OFF DRN-projecting RGCs from control (rod/cone intact, $n=10$ cells) and MNU-treated (rodless/coneless, $n=10$ cells) animals. One-way ANOVA; *** $<0.00 \mathrm{I}$. (d) Forced swimming test (FST) of MNU-treated animals after intravitreal saline injection (MNU-DD-VEH-I) or $6 \mu \mathrm{M}$ TTX (MNU-DD-TTX). TTX increased immobile score and climbing score and reduced swimming score. One-way ANOVA, Tukey's post-hoc test; *** $p<0.001$.

\section{Specific Ablation of DRN-projecting RGCs Alters Depressive-like Behavior}

To attribute the anti-depressive-like effect of silencing RGCs specifically to DRN-projecting RGCs, we developed a novel saporin-based immunotoxin, anti- CTB conjugated to saporin (anti-CTB-SAP; Figure 6). To test this new compound, SC-projecting RGCs were retrogradely labeled by unilateral injection of CTB into the SC $(n=16)$ (Figure 6). Three days later, animals received bilateral intravitreal injections of either saline $(n=4)$ or antiCTB-SAP at different dosages $(0.5 \mu \mathrm{g} / \mathrm{eye}, 2 \mu \mathrm{g} / \mathrm{eye}$ and $4 \mu \mathrm{g}$ /eye, $n=4$ /group). After treatment, the number of CTBlabeled RGCs was counted in a standardized area in the region of peak density in each retina. After images were captured for quantification of SC-projecting RGCs, the retinas were processed further for immunocytochemical localization of melanopsin, the opsin expressed in ipRGCs (Hattar et al, 2002). The results indicate that the antiCTB-saporin immunotoxin selectively ablates CTB-labeled RGCs in a dose-dependent manner (one-way ANOVA, $\left.\mathrm{F}_{3,12}=341.94, \quad p<0.001\right) \quad$ while sparing most nonCTB-labeled melanopsin-expressing ipRGCs (one-way ANOVA, $\mathrm{F}_{3,12}=0.29, p=0.835$ ) (Figure 6a-c). Furthermore, we investigated whether ipRGCs had a role in regulating behavior in the FST by selectively eliminating melanopsin-expressing ipRGCs with an anti-melanopsinsaporin immunotoxin. As shown in Figure 7, immunotoxin ablation of $\approx 70 \%$ of melanopsin-expressing ipRGCs did not affect FST-associated behaviors in MNU-DD animals.

To investigate the effects of selective immunotoxin ablation of DRN-projecting RGCs on behavioral state, CTB was injected into the DRN of MNU-treated animals that subsequently received intravitreal injection of anti-CTB-SAP. As shown in Figure $8 \mathrm{a}-\mathrm{c}$, a large majority (68\%) of retrogradely labeled DRN-projecting RGCs was selectively eliminated by the immunotoxin (one-way ANOVA, $\left.\mathrm{F}_{1,14}=55.18, p<0.001\right)$. The significant reduction in DRN-projecting RGCs resulted in a change in behavioral state: immobile score was significantly increased (one-way ANOVA, $\mathrm{F}_{1,10}=24.05 ; p<0.001$ ), whereas swimming was significantly reduced (one-way ANOVA, $\mathrm{F}_{1,10}=259.92$, $p<0.001$; Figure 8d; Supplementary Movie S4). Using this novel immunotoxin approach, the data indicate that selective reduction of DRN-projecting RGCs can reverse the anti-depressive-like behavior generated by MNU treatment in DD animals.

The impact of selective immunotoxin elimination of DRN-projecting RGCs on behavioral state described above was demonstrated in MNU-treated animals. However, it is possible that the immunotoxin could non-selectively injure or destroy photoreceptors. To test this possibility, 

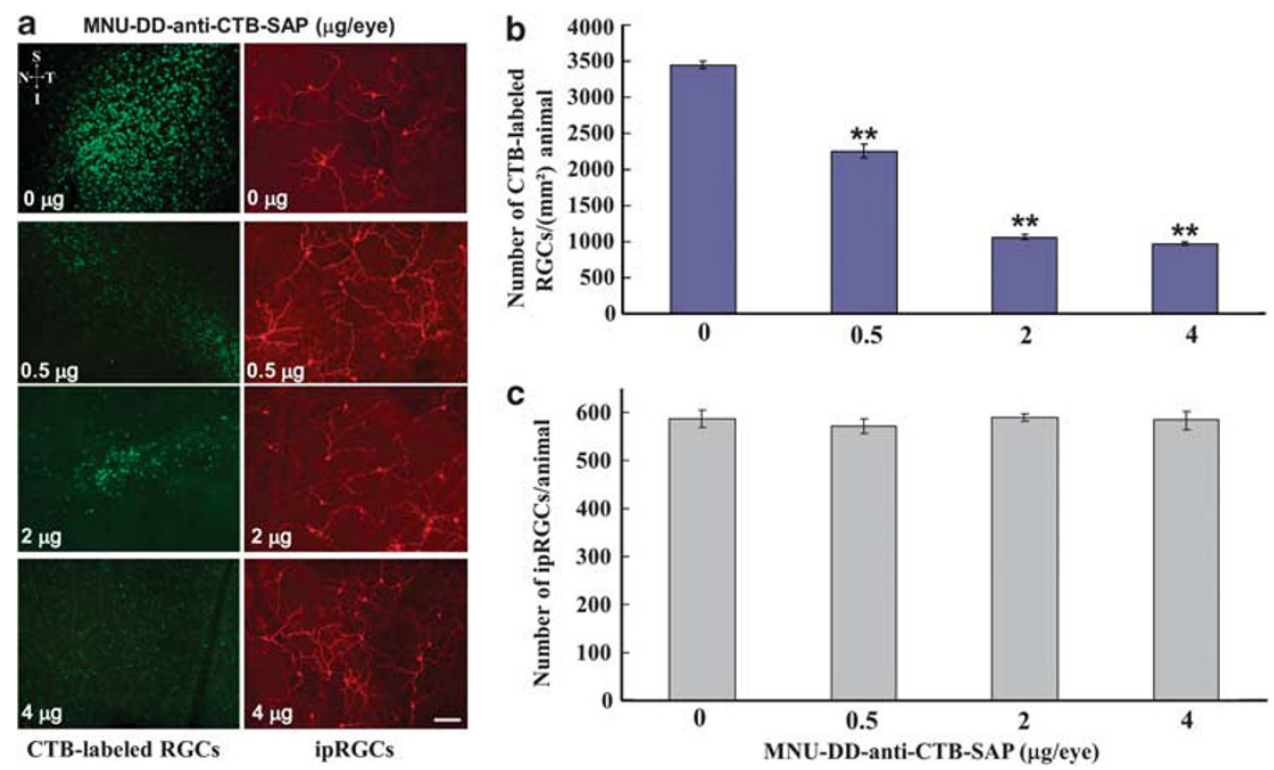

Figure 6 Anti-cholera toxin subunit B-saporin (Anti-CTB-saporin) immunotoxin selectively eliminates CTB-labeled retinal ganglion cells (RGCs) projecting to the superior colliculus (SC). SC-projecting RGCs were retrogradely labeled by unilateral injection of CTB into the SC and 3 days later the eye contralateral to the injected SC received an intravitreal injection of saline $(5 \mu \mathrm{l})$ or anti-CTB-saporin $(0.5-4 \mu \mathrm{g}$ in $5 \mu \mathrm{l}$ saline). After 7 days, the retinas were examined for CTB-labeled SC-projecting RGCs. (a) Images in left column demonstrate the retinal region exhibiting the greatest density of CTB-labeled RGCs (where the number of cells was quantified) in animals injected with vehicle or anti-CTB-saporin. After images were captured for quantification of SC-projecting RGCs, the retinas were processed further for immunocytochemical localization of melanopsin, the opsin expressed in intrinsically light-sensitive RGCs (ipRGCs; Hattar et al, 2002); all melanopsin-labeled RGCs were counted in each retina. Images in right column illustrate melanopsinexpressing RGCs following saline or anti-CTB-saporin application. Retina orientation: S: Superior, I: Inferior, N: Nasal, and T: Temporal. (b) Quantification of the number of CTB-labeled SC-projecting RGCs in saline and immunotoxin-injected eyes demonstrating that the anti-CTB-saporin eliminated CTB-labeled RGCs in a dose-dependent manner (4 retinas/group, one-way ANONA; *** $<0.00 \mathrm{I}$ ). (c) In the same retinas in which anti-CTB-saporin ablated CTBlabeled RGCs, the immunotoxin had no effect on the number of ipRGCs observed. The results indicate that the anti-CTB-saporin immunotoxin selectively ablates CTB-labeled RGCs while sparing non-CTB-labeled (melanopsin) RGCs. The $2.0 \mu \mathrm{g} /$ eye was selected for further experiments. Bar $=100 \mu \mathrm{m}$.
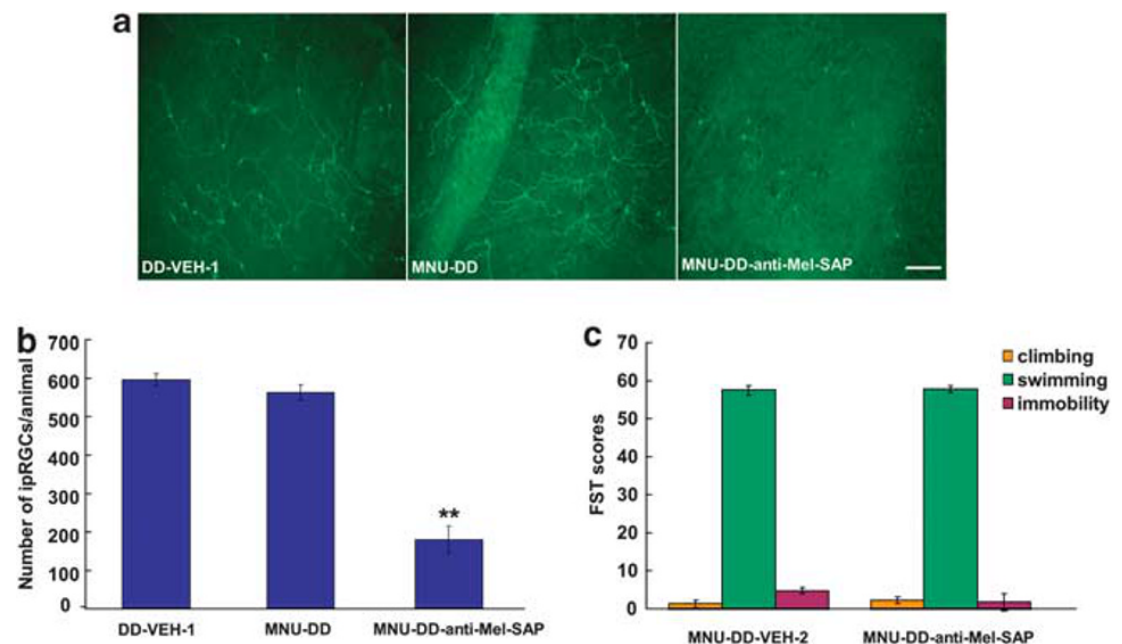

Figure 7 Immunotoxin ablation of melanopsin-expressing intrinsically photosensitive retinal ganglion cells (ipRGCs) does not affect forced swimming test (FST). (a) Images of melanopsin-expressing ipRGCs in a retinal whole mount from DD and MNU-DD animals receiving intravitreal injection of vehicle (left and middle) or anti-melanopsin-saporin immunotoxin (anti-Mel-SAP; right). Bar = $100 \mu \mathrm{m}$. (b) Quantification of melanopsin-expressing ipRGCs in animals maintained in DD receiving intravitreal saline (DD-VEH-I), treated with MNU and maintained in DD and receiving intravitreal saline (MNU-DD-VEH-2) and MNU-treated animals receiving intravitreal immunotoxin anti-melanopsin-saporin (MNU-DD-anti-Mel-SAP), DD-VEH-I group serves as control. ( $n=6 /$ group, one-way ANOVA; $* * * 0.001$ ). The immunotoxin anti-melanopsin-saporin injected into the vitreous chamber of the eye specifically ablated melanopsinexpressing RGCs with minimal effect on non-melanopsin-expressing RGCs (Göz et al, 2008; Hatori et al, 2008). (c) There were no significant changes in FST associated behaviors in MNU-DD-anti-Mel-SAP animals compared with MNU-DD-VEH-2 group ( $n=6 /$ group, one-way ANOVA; $p=0.6$ I8).

DRN-projecting RGCs were selectively abolished with immunotoxin in animals with intact photoreceptors. As shown in Figure 8e, specific ablation of DRN-projecting RGCs in animals with intact retinas significantly increased immobile (one-way ANOVA, $\mathrm{F}_{1,10}=8.62, p<0.05$ ) and climbing scores (One-way ANOVA, $\mathrm{F}_{1,10}=14.69, p<0.05$ ) while significantly decreasing swimming score compared with vehicle-treated controls (LD-VEH; one-way ANOVA, 
a

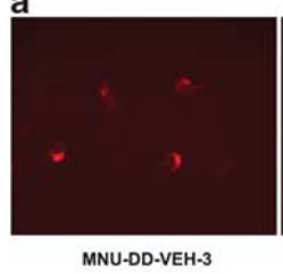

C

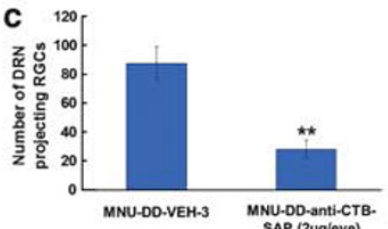

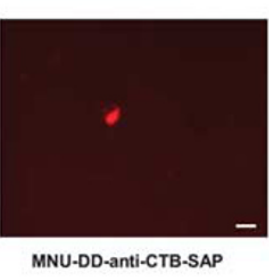

b

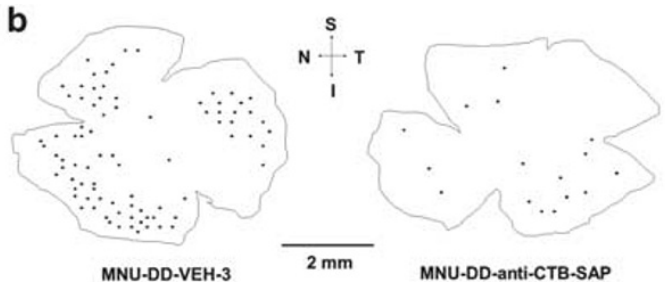

Figure 8 Specific ablation of dorsal raphe nucleus-projecting retinal ganglion cells (DRN-projecting RGCs) alters depressive-like behavior. (a) Examples of retrogradely labeled DRN-projecting RGCs in retinal whole mounts after cholera toxin subunit B (CTB) injection into DRN and then after intravitreal (left) saline injection or (right) anti-CTB-saporin (anti-CTB-SAP) immunotoxin $(2 \mu \mathrm{g} / \mathrm{eye})$ injection. Bar $=20 \mu \mathrm{m}$. (b) Distribution of CTB-labeled DRN-projecting RGCs in retinal whole mounts in photoreceptor ablation-continuous darkness (MNU-DD)-treated animals 7 days after intravitreal vehicle (left) or anti-CTBSAP immunotoxin (2 $\mu \mathrm{g} /$ eye; right). Retina orientation: S: Superior, I: Inferior, N: Nasal, and T: Temporal. (c) Quantification of DRN-projecting RGCs in saline (MNU-DD-VEH-3) and immunotoxin-treated eyes from MNU-DD animals illustrating significant reduction in the number of CTB-labeled RGCs (87.7

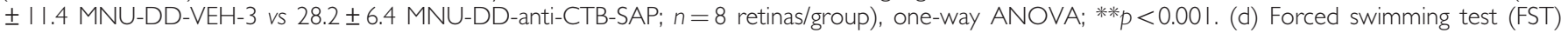
scores in MNU-DD animals after CTB injection into the DRN followed by intravitreal injection of vehicle or immunotoxin. Specific ablation of DRNprojecting RGCs (MNU-DD-anti-CTB-SAP) significantly increased immobile and climbing scores, whereas decreasing swimming score compared with controls. One-way ANOVA; *** <0.00I. (e) FST climbing, swimming, and immobility scores at Zeitgeber Time 6 for animals housed in LD conditions treated with saline and anti-CTB-SAP. Specific ablation of DRN-projecting RGCs (LD-anti-CTB-SAP) significantly increased immobile and climbing scores, whereas decreasing swimming score. LD-VEH group serves as control. One-way ANOVA; * $p<0.05$; ** $p<0.00$ I.

$\left.\mathrm{F}_{1,10}=50.44, p<0.001\right)$. These results are consistent with the interpretation that DRN-projecting RGCs modulate behavioral state.

The results from the TST paralleled the robust effects observed with the FST. DD animals had significantly increased immobile time compared with LD animals (one-way ANOVA, $\quad \mathrm{F}_{1,10}=463.12, \quad p<0.001$ ) and MNU treatment significantly reduced immobile time in MNU-DD compared with DD animals (one-way ANOVA, $\mathrm{F}_{1,10}=889.22, p<0.001$; Figure 2a). Conversely, MNU-DD animals receiving intravitreal injection of anti-CTB-SAP (one-way ANOVA, $\mathrm{F}_{1,10}=79.72, p<0.001$ ) or TTX (one-way ANOVA, $\left.\mathrm{F}_{1,10}=78.56, p<0.001\right)$ showed greatly increased immobile times (Figure 2b). These findings are similar to the results obtained in the FST.

As shown in Figure 3a, maintain animals in DD was associated with a significant decrease in sucrose preference compared with animals maintained in LD conditions (one-way ANOVA, $\mathrm{F}_{1,10}=9.26, p<0.05$ ). Consistent with the results from the other behavioral tests, MNU treatment was anti-depressive, significantly increasing sucrose preference compared with DD animals (one-way ANOVA, $\mathrm{F}_{1,10}=71.23, p<0.001$ ), whereas selective removal of DRNprojecting RGCs in MNU-DD animals by immunotoxin significantly decreased sucrose preference compared with MNU-DD-VEH-3 animals (one-way ANOVA, $\mathrm{F}_{1,10}=21.44$, $p<0.01)$. Taken together, the findings demonstrate that DRN-projecting RGCs have an important role in regulating behavioral state.

\section{Relationship between DRN 5-HT-positive Neurons and FST Behaviors}

Next, we investigated DRN serotonergic tone using immunocytochemical staining for 5-HT in a subset of animals $(n=4)$ from each group. The number of 5-HTpositive $(+)$ neurons in the dorsal and lateral divisions of the DRN varied significantly among experimental groups (one-way ANOVA, dorsal division: $\mathrm{F}_{5,18}=123.92, p<0.001$; lateral division: $\left.\mathrm{F}_{5,18}=101.63, p<0.001\right)$; conditions that increased RGC activity (MNU-DD) increased the number of DRN 5-HT neurons and conversely, conditions that decreased RGC activity (DD, MNU-DD-TTX; MNUDD-SAP) decreased the number of DRN 5-HT + neurons relative to levels in animals housed in $\mathrm{LD}$ conditions (one-way ANOVA, MNU-DD vs LD: $\mathrm{F}_{1,6}=156.31, p<0.001$; $\mathrm{DD}$ vs LD: $\mathrm{F}_{1,6}=77.25, p<0.001$; MNU-DD-TTX $v s$ LD: $\mathrm{F}_{1,6}=18.98, p<0.01$; MNU-DD-SAP $v s$ LD: $\mathrm{F}_{1,6}=18.21$, $p<0.01)$. These effects were more prominent in the lateral DRN (MNU-DD increased lateral 5-HT + neurons 12-fold over DD but only sevenfold over DD in the dorsal DRN; Figure 9a-b). Chronic treatment of DD animals with the SSRI anti-depressant fluoxetine (DD-FLX) significantly reduced immobile scores in the FST (one-way ANOVA, $\mathrm{F}_{1,10}=92.80, \quad p<0.001$ ) and TST (one-way ANOVA, $\mathrm{F}_{1,10}=112.97, p<0.001$; Figure $2 \mathrm{c}-\mathrm{d}$ and Supplementary Movie S5) and significantly elevated 5-HT levels over DD conditions (Figure 9b). DD animals treated for 21 days with imipramine, a non-SSRI tricyclic anti-depressant (DD-IMI), also reversed the depressive-like behavior evoked by DD (FST: one-way ANOVA, $\mathrm{F}_{1,10}=84.24, p<0.001$; TST: one-way ANOVA, $\mathrm{F}_{1,10}=84.31, p<0.001$ ), as illustrated in Figure $2 \mathrm{c}-\mathrm{d}$. Fluoxetine and imipramine also reversed the reduced sucrose preference behavior evoked by DD (one- way ANOVA, $\mathrm{F}_{2,12}=5.75, p<0.05$; Turkey's post hoc comparison indicated that sucrose preference of DD-VEH-2 group is significantly lower than that in DDFLX $(p<0.05)$ and DD-IMI group $(p<0.05))$ (Figure 9b). As revealed in Figure $2 c$, the impacts of the two anti-depressants are different on FST behavior; this could 
a
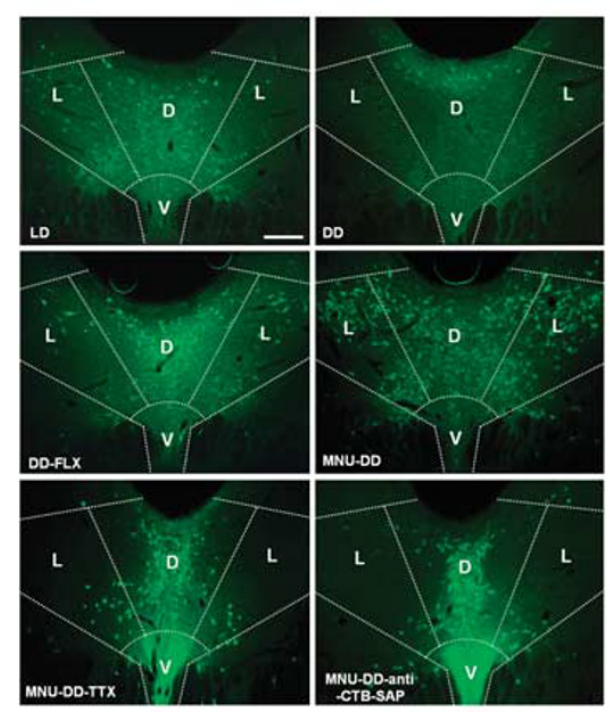
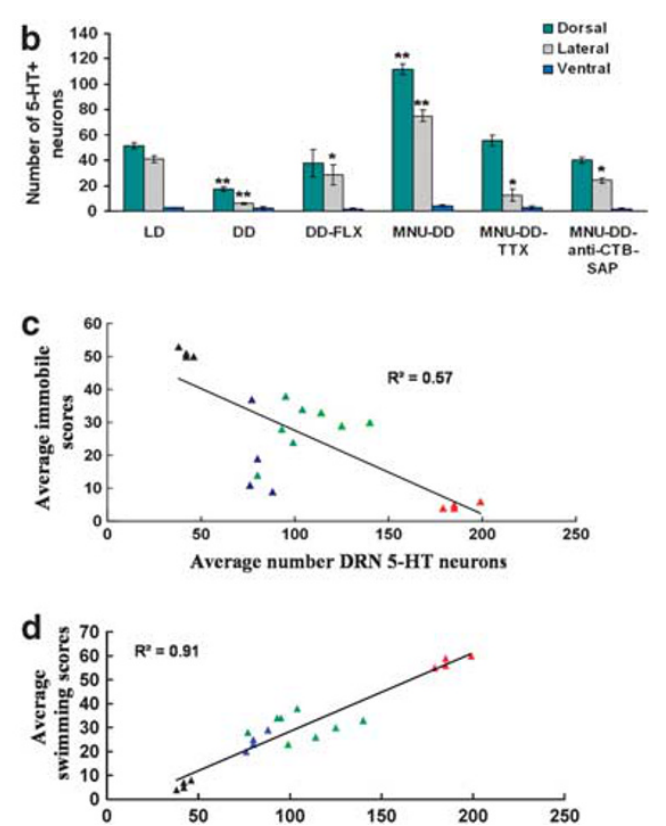

Figure 9 Relationship between dorsal raphe nucleus 5-hydroxytrytamine positive (DRN 5-HT +) neurons and forced swimming test (FST)-associated behaviors. (a) Images of immunocytochemically labeled 5-HT expressing neurons in dorsal (D), lateral (L), and ventral $(V)$ DRN regions of the I2-h light, I2-h dark (LD), continuous darkness (DD), DD-fluoxetine (DD-FLX), photoreceptor ablation-DD (MNU-DD), MNU-DD-tetrodotoxin (MNU-DD-TTX), and MNU-DD-anti-cholera toxin subunit B saporin immunotoxin (MNU-DD-anti-CTB-SAP) groups. Bar $=200 \mu \mathrm{m}$. (b) Quantification of 5-HT + neurons in DRN subdivisions, $n=4$ animals/group (mean \pm SEM). LD group serves as control. One-way ANOVA; * $p<0.05$; ** $p<0.00$ I. (c, d) Regression analysis of

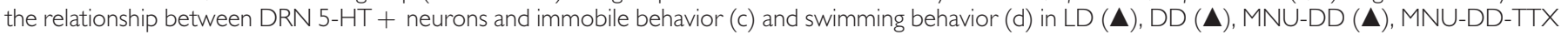
$(\mathbf{\Delta})$ and MNU-DD-SAP $(\mathbf{\Delta})$

be due to their targeting of distinct systems (Detke et al, 1996).

There was a strong negative correlation between DRN 5 -HT + neurons and immobility $\left(r^{2}=0.57\right.$; Figure 9c). Swimming was also highly correlated with DRN 5-HT + cells $\left(r^{2}=0.91\right.$; Figure 9d), whereas climbing was not $\left(r^{2}=0.13 ;\right.$ Supplementary Figure S1). The correlations between swimming and immobility score with DRN $5-\mathrm{HT}+$ neurons in the lateral and dorsal subdivisions were similar (Supplementary Figures S2 and S3). The results demonstrate that RGC input to the DRN significantly impacts DRN 5-HT tone which, in turn, has a critical role in regulating behavioral state.

\section{DISCUSSION}

Light is a potent modulator of affective behavior; light treatment is anti-depressive (Golden et al, 2005) and light deprivation induces depressive-like behaviors (current study; Gonzalez and Aston-Jones, 2008; Monje et al, 2011; Lau et al, 2011). The present evidence indicates that RGCs with alpha-like morphological and Y-like physiological properties convey signals to the DRN and modulate serotonin levels. Silencing RGC firing or specific immunotoxin ablation of DRN-projecting RGCs increased depressive-like behavior and reduced serotonin levels in the DRN. Together, this study demonstrates that retino-raphe signals modulate DRN serotonergic tone and affective behavior.

Alpha/Y-like RGCs are a common cell type in the mammalian retina that innervate target structures in the brain that process visual information. These cells receive visual inputs from both rod and cone photoreceptors but rod input predominates, and it is thought that they serve a visual alerting function, although their precise role in visual perception remains ill-defined (Peichl et al, 1987; Peichl, 2005). Y-type RGC physiology is well characterized. The ON type $\mathrm{Y}$ cell gives a brisk transient discharge at the onset of a light stimulus within its receptive field center, whereas the OFF Y cell has a similar type of response at the offset of a light stimulus and these cells are characterized by integration of stimuli using non-linear spatial summation (Cleland and Levick, 1974; Enroth-Cugell and Robson, 1966). DRN-projecting RGCs have been described in numerous species, including primates, cats, and rodents (Foote et al, 1978; Fite et al, 1999; Reuss and Fuchs, 2000; Luan et al, 2011). However, the DRN is not a component of the image-forming visual system, and therefore it might have been expected that the DRN would receive retinal signals from melanopsin-expressing ipRGCs, the primary RGC type that contributes to non-image-forming visual functions (Pickard and Sollars, 2012). This, however, does not appear to be the case as DRN-projecting alpha RGCs display canonical ON or OFF Y-type RGC physiological properties (ie, brisk transient responses and non-linear spatial summation) and neither melanopsin photopigment nor an intrinsic photoresponse has been detected in these RGCs (Luan et al, 2011). A very recent study has shown, however, that an ON alpha/Y-like RGC (the M4 ipRGC cell type) projects to the dorsal lateral geniculate nucleus (dLGN), the primary thalamic nucleus associated with visual perception, expresses very low levels of melanopsin, 
and exhibits a weak intrinsic photoresponse (Estevez et al, 2012). Specialized immunocytochemical amplification methods were necessary to detect melanopsin in these cells and even then not all physiologically confirmed M4 ipRGCs were melanopsin-positive (Estevez et al, 2012). It is likely that the standard immunocytochemical protocol used to label melanopsin RGCs (Luan et al, 2011) would have been insufficient to detect melanopsin in DRN-projecting RGCs if these cells are M4 ipRGCs. Although it cannot be said with certainty that the ON alpha/Y-like RGCs that innervate the DRN are different from the M4 ipRGCs described by Estevez et al (2012), their brisk transient response to photic stimuli differs from the tonic-sustained responses of M4 cells and, more importantly, Estevez et al (2012) did not observe any OFF alpha/Y-like ipRGCs expressing melanopsin. In the DD-maintained MNU-treated animals used as a model in the present study, OFF cells in particular had a crucial role in altering behavioral mood as it was the spontaneous activity of OFF RGCs only that increased dramatically following MNU treatment.

Under normal conditions, the DRN receives input from both ON and OFF Y-type RGCs (Luan et al, 2011) and their maintained firing frequency is dependent on the luminance level (Barlow and Levick, 1969; Troy and Robson, 1992). Under DD conditions, both ON and OFF Y-type cells cease light-evoked responses but maintain a low spontaneous firing rate (Figure $5 \mathrm{c}$ ). After MNU-induced photoreceptor depletion, OFF Y-type cells subsequently showed a sixfold increase in spontaneous firing rate, which, in turn, improved affective behavior. Although a relatively small number of alpha/Y-like RGCs innervates the DRN, because these RGCs have a broad dendritic field (Luan et al, 2011), only $\approx 700$ are necessary to tile the entire retinal surface (Supplementary Table S1). All alpha/Y-like RGCs have relatively large diameter axons; perhaps the high conduction velocity of these RGCs (Peichl et al, 1987) is the salient component of the visual signal being conveyed to the DRN (Chen and Regehr, 2003).

The findings in the present study showed that the level of neural activity of DRN-projecting RGCs was directly related to behavioral state. Moreover, DRN-projecting RGC activity was correlated with DRN 5-HT levels and affective behavior; MNU-DD animals contained the greatest number of DRN 5-HT + neurons, whereas DD animals had the fewest. Reducing RGC signals to the DRN in MNU-DD animals, either by acute TTX inhibition of firing or by immunotoxin ablation of DRN-projecting RGCs, decreased DRN 5-HT levels and significantly elevated behavioral indices of depressive-like behavior. Chronic inhibition of DRNprojecting RGCs or more complete immunotoxin lesioning of DRN-projecting RGCs in MNU-DD animals would likely have produced even greater effects on DRN 5-HT tone and depressive-like behavior.

We also examined whether the SSRI anti-depressant fluoxetine would improve the depressive-like behavior evoked by DD treatment in a manner similar to the MNUevoked increase in DRN-projecting RGC spontaneous activity. Indeed, as shown in Figure 2c and Supplementary Movie S5, chronic fluoxetine treatment significantly reduced immobile score, although elevating swimming while having no effect on climbing behavior in the FST. This result is consistent with previous reports that SSRIs are both anti-depressive and elevate swimming without affecting climbing behavior in the FST (Lucki, 1997) and similar to the effects observed by MNU treatment of DD animals. Thus, the depressive-like behavior induced by light deprivation was alleviated both by fluoxetine and increased activity of DRN-projecting RGCs. Importantly as shown previously (Lucki, 1997), fluoxetine effects in this study were specific, in that swimming time in the FST was elevated without an increase in general locomotor activity. Similarly, the anti-depressant effects of MNU treatment did not generate an increase in locomotor activity, in agreement with the lack of MNU-evoked increased locomotor activity reported by Boudard et al (2009). Moreover, DD animals treated with imipramine, a non-SSRI tricyclic anti-depressant, also showed a reversal in depressive-like behavior in the FST and TST without affecting the locomotor activity in the OFT. DRN 5-HT levels were also highly correlated with FST swimming but not climbing behavior. Collectively, the data support the view that increased retinal input to the DRN is anti-depressive.

We describe a non-pharmacological anti-depressant treatment, activation of direct retino-raphe input to the DRN that modulates serotonergic tone and depressive-like behavior. This novel result sheds new light on our current understanding of affective visual information processing.

\section{ACKNOWLEDGEMENTS}

This work was supported by National Basic Research Program (2011CB510206 and 2009CB320901 to MP); NSFC grants (31170986 and 30831160516 to MP), Research Scheme under the contract N_HKU750/08 (KFS), the Fundamental Research Funds for the Central Universities (21609101, KFS); National Basic Research Program of China (973 Program) (2011CB707501, KFS), and the USA National Institutes of Health grants EY 017809 and NS 077003 (GEP).

This work was supported by the government grants from China, Hong Kong, and the United States.

\section{DISCLOSURE}

The authors declare no conflict of interest.

\section{REFERENCES}

Barlow HB, Levick WR (1969). Changes in the maintained discharge with adaptation level in the cat retina. J Physiol 202: 699-718.

Bijak M, Papp M (1995). The effect of chronic treatment with imipramine on the responsiveness of hippocampal CA1 neurons to phenylephrine and serotonin in a chronic mild stress model of depression. Eur Neuropsychopharmacol 5: 43-48.

Boudard DL, Mendoza J, Hicks D (2009). Loss of photic entrainment at low illuminances in rats with acute photoreceptor degeneration. Eur J Neurosci 30: 1527-1536.

Chen C, Regehr WG (2003). Presynaptic modulation of the retinogeniculate synapse. J Neurosci 23: 3130-3135.

Cleland BG, Levick WR (1974). Brisk and sluggish concentrically organized ganglion cells in the cat's retina. J Physiol 240: 421-456.

Cryan JF, Valentino RJ, Lucki I (2005a). Assessing substrates underlying the behavioral effects of antidepressants using the 
modified rat forced swimming test. Neurosci Biobehav Rev 29: 547-569.

Detke MJ, Rickels M, Lucki I (1996). Active behaviors in the rat forced swimming test differentially produced by serotonergic and noradrenergic antidepressants. Psychopharmacology (Berl) 121: 66-72.

Edwards JA, Cline HT (1999). Light-induced calcium influx into retinal axons is regulated by presynaptic. J Neurophysiol 81: 895-907.

Enroth-Cugell C, Robson JG (1966). The contrast sensitivity of retinal ganglion cells of the cat. J Physiol (Lond) 187: 517-552.

Estevez ME, Fogerson PM, Ilardi MC, Borghuis BG, Chan E, Weng $\mathrm{S}$ et al (2012). Form and function of the $\mathrm{m} 4$ cell, an intrinsically photosensitive retinal ganglion cell type contributing to geniculocortical vision. J Neurosci 32: 13608-13620.

Fite KV, Janusonis S, Foote W, Bengston L (1999). Retinal afferents to the dorsal raphe nucleus in rats and Mongolian gerbils. J Comp Neurol 414: 469-484.

Foote WE, Taber-Pierce E, Edwards L (1978). Evidence for a retinal projection to the midbrain raphe of the cat. Brain Res 156: 135-140.

Golden RN, Gaynes BN, Ekstrom RD, Hamer RM, Jacobsen FM, Suppes $\mathrm{T}$ et al (2005). The efficacy of light therapy in the treatment of mood disorders: a review and meta-analysis of the evidence. Am J Psychiatry 162: 656-662.

Gonzalez MM, Aston-Jones G (2008). Light deprivation damages monoamine neurons and produces a depressive behavioral phenotype in rats. Proc Natl Acad Sci USA 105: 4898-4903.

Göz D, Studholme K, Lappi DA, Rollag MD, Provencio I, Morin LP (2008). Targeted destruction of photosensitive retinal ganglion cells with a saporin conjugate alters the effects of light on mouse circadian rhythms. PLoS One 3: e3153.

Güler AD, Ecker JL, Lall GS, Haq S, Altimus CM, Liao HW et al (2008). Melanopsin cells are the principal conduits for rod-cone input to non-image-forming vision. Nature 453: 102-105.

Hatori M, Le H, Vollmers C, Keding SR, Tanaka N, Buch T et al (2008). Inducible ablation of melanopsin-expressing retinal ganglion cells reveals their central role in non-image forming visual responses. PLoS One 3: e2451.

Hattar S, Liao HW, Takao M, Berson DM, Yau KW (2002). Melanopsin-containing retinal ganglion cells: architecture, projections, and intrinsic photosensitivity. Science 295: 1065-1070.

Herrold KM (1967). Pigmentary degeneration of the retina induced by $N$-methyl- $N$-nitrosourea. An experimental study in Syrian hamsters. Arch Ophthalmol 78: 650-653.

Lau BW, Ren C, Yang J, Yan SW, Chang RC, Pu M et al (2011). Light deprivation induces depression-like behavior and suppresses neurogenesis in diurnal mongolian gerbil (Meriones unguiculatus). Cell Transplant 20: 871-881.

Lewy AJ, Sack RL, Miller LS, Hoban TM (1987). Antidepressant and circadian phase-shifting effects of light. Science 235: 352-354.

Luan L, Ren C, Lau BW, Yang J, Pickard GE, So KF et al (2011). Y-like retinal ganglion cells innervate the dorsal raphe nucleus in the Mongolian gerbil (Meriones unguiculatus). PLoS One 6: e18938.

Lucki I (1997). The forced swimming test as a model for core and component behavioral effects of antidepressant drugs. Behav Pharmacol 8: 523-532.

Machado DG, Cunha MP, Neis VB, Balen GO, Colla A, Grando J et al (2012). Fluoxetine reverses depressive-like behaviors and increases hippocampal acetylcholinesterase activity induced by olfactory bulbectomy. Pharmacol Biochem Behav 103: $220-229$.

Margolis DJ, Detwiler PB (2007). Different mechanisms generate maintained activity in ON and OFF retinal ganglion cells. J Neurosci 27: 5994-6005.
Monje FJ, Cabatic M, Divisch I, Kim EJ, Herkner KR, Binder BR et al (2011). Constant darkness induces IL-6-dependent depression-like behavior through the NF- $\kappa \mathrm{B}$ signaling pathway. J Neurosci 31: 9075-9083.

Monteggia LM, Luikart B, Barrot M, Theobold D, Malkovska I, Nef S et al (2007). Brain-derived neurotrophic factor conditional knockouts show gender differences in depression-related behaviors. Biol Psychiatry 61: 187-197.

Page ME, Detke MJ, Dalvi A, Kirby LG, Lucki I (1999). Serotonergic mediation of the effects of fluoxetine, but not desipramine, in the rat forced swimming test. Psychopharmacology (Berl) 147: $162-167$.

Paxinos G, Watson C (1998). The Rat Brain in Stereotaxic Coordinates. 4 th edn.

Peichl L (2005). Diversity of mammalian photoreceptor properties: adaptations to habitat and lifestyle? Anat Rec A Discov Mol Cell Evol Biol 287: 1001-1012.

Peichl L, Ott H, Boycott BB (1987). Alpha ganglion cells in mammalian retinae. Proc $R$ Soc Lond B Biol Sci 231: 169-197.

Pickard GE, Sollars PJ (2012). Intrinsically photosensitive retinal ganglion cells. Rev Physiol Biochem Pharmacol 162: 59-90.

Pickles AR, Hagan JJ, Jones DN, Hendrie CA (2012). Short-term individual housing induced social deficits in female Mongolian gerbils: attenuation by chronic but not acute imipramine. Neuropharmacology 62: 1993-1998.

$\mathrm{Pu}$ M (1999). Dendritic morphology of cat retinalganglion cells projecting to suprachiasmatic nucleus. J Comp Neurol 414: 267-274.

$\mathrm{Pu}$ M, Xu L, Zhang H (2006). Visual response properties of retinal ganglion cells in the royal college of surgeons dystrophic rat. Invest Ophthalmol Vis Sci 47: 3579-3585.

Reuss S, Fuchs E (2000). Anterograde tracing of retinal afferents to the tree shrew hypothalamus and raphe. Brain Res 874: 66-74.

Rodríguez-Gaztelumendi A, Rojo ML, Pazos A, Díaz A (2009). Altered $\mathrm{CB}$ receptor-signaling in prefrontal cortex from an animal model of depression is reversed by chronic fluoxetine. J Neurochem 108: 1423-1433.

Steru L, Chermat R, Thierry B, Simon P (1985). The tail suspension test: a new method for screening antidepressants in mice. Psychopharmacol (Berl) 85: 367-370.

Svenningsson P, Tzavara ET, Witkin JM, Fienberg AA, Nomikos GG, Greengard P (2002). Involvement of striatal and extrastriatal DARPP-32 in biochemical and behavioral effects of fluoxetine (Prozac). Proc Natl Acad Sci USA 99: 3182-3187.

Troy JB, Robson JG (1992). Steady discharges of X and Y retinal ganglion cells of cat under photopic illuminance. Vis Neurosci 9: 535-553.

Vandewalle G, Maquet P, Dijk DJ (2009). Light as a modulator of cognitive brain function. Trends Cog Sci 13: 429-438.

Varty GB, Cohen-Williams ME, Hunter JC (2003). The antidepressant-like effects of neurokinin NK1 receptor antagonists in a gerbil tail suspension test. Behav Pharmacol 14: 87-95.

Viswanathan S, Frishman LJ, Robson JG (2000). The uniform field and pattern ERG in macaques with experimental glaucoma: removal of spiking activity. Invest Ophthalmol Vis Sci 41: 2797-2810.

Warthen DM, Wiltgen BJ, Provencio I (2011). Light enhances learned fear. Proc Natl Acad Sci USA 108: 13788-13793.

Wenzel A, Grimm C, Marti A, Kueng-Hitz N, Hafezi F, Niemeyer G et al (2000). C-fos controls the 'private pathway' of light-induced apoptosis of retinal photoreceptors. J Neurosci 20: 81-88.

Wilson N (2002). Depression and its relation to light deprivation. Psychoanal Rev 89: 557-567.

Wirz-Justice A, Benedetti F, Terman M (2009). Chronotherapeutics for Affective Disorders. Karger: New York. 
Witkovsky P, Veisenberger E, Haycock JW, Akopian A, Garcia-Espana A, Meller E (2004). Activity-dependent phosphorylation of tyrosine hydroxylase in dopaminergic neurons of the rat retina. J Neurosci 24: 4242-4249.

Yau KW, Hardie RC (2009). Phototransduction motifs and variations. Cell 139: 246-264.

Yoshizawa K, Tsubura A (2005). Characteristics of $\mathrm{N}$-methyl- $\mathrm{N}$ nitrosourea-induced retinal degeneration in animals and application for the therapy of human retinitis pigmentosa. Nihon Ganka Gakkai Zasshi 109: 327-337.

(c) (1) (-) $\odot$ This work is licensed under a Creative Commons Attribution-NonCommercial-NoDerivs 3.0 Unported License. To view a copy of this license, visit http:// creativecommons.org/licenses/by-nc-nd/3.0/

Supplementary Information accompanies the paper on the Neuropsychopharmacology website (http://www.nature.com/npp) 